\title{
Blood and iron
}

\author{
Friedrich C. Luft $^{1}$
}

Published online: 17 April 2015

(C) Springer-Verlag Berlin Heidelberg 2015

"Not through speeches and majority decisions" will the mechanisms of hemochromatosis be decided, but through "blood and iron"-based scientific research. An absolute requirement for most organisms, iron is essential to life. However, its catalytic activity converting hydrogen peroxide into free radicals can cause damage to most cellular structures leading to death. The average human contains about $5 \mathrm{~g}$ of iron. Half is contained in erythrocytes bound to hemoglobin. Most of the rest is bound to ferritin complexes in all cells but most commonly in the bone marrow, liver, and spleen. Iron is not excreted. The egress involves blood loss, cell shedding, and (some) sweating [1]. The wonder of iron metabolism has received considerable attention and for good reason. Iron deficiency is the commonest disease worldwide, while iron overload, such as hemochromatosis, is by no means rare.

The entry portal of dietary iron is the enterocyte in the duodenum; first, a ferric-reductase enzyme, duodenal cytochrome B (Dcytb), reduces the poorly soluble and absorbable $\mathrm{Fe}^{3+}$ to the $\mathrm{Fe}^{2+}$ form. Then, the divalent metal transporter 1 (DMT1) transports iron across the enterocyte's cell membrane [2]. The $\mathrm{Fe}^{2+}$ can be reconverted to $\mathrm{Fe}^{3+}$ and bound to apoferritin, in which case the iron is trapped and stored as ferritin within the enterocytes until the cell is sloughed off into feces. Alternatively, the iron can be transferred into the circulatory system through a protein called ferroportin. Hephaestin, a ferroxidase that oxidizes $\mathrm{Fe}^{2+}$ to $\mathrm{Fe}^{3+}$ and resides mainly in the small intestine, supports ferroportin to transport iron across the basolateral end of intestinal cells to the bloodstream

Friedrich C. Luft

friedrich.luft@charite.de

1 Experimental and Clinical Research Center, Charité Medical Faculty, Berlin, Germany
[3]. Hepcidin, encoded by the gene $H A M P$, is an important negative regulator of iron efflux. Two forms exist; one is 20 and the other is 25 amino acids in length. Hepcidin 25 binds to ferroportin in enterocytes and macrophages leading to lysosomal degradation. Thereby, dietary iron absorption is turned off effectively [4]. Expression and secretion of hepcidin are regulated in the liver involving iron sensing at the membrane and Bmp/Smad signaling (Fig. 1) [5]. Overall, iron absorption is well regulated in the body. However, for example, in patients with iron-deficiency anemia or in the presence of inflammation, iron key players are dysregulated [4].

Much has been learned from genetic disease of iron overload [5]. One of the defective genes responsible for the most common type of hereditary hemochromatosis is the HFE gene, which encodes for the human hemochromatosis protein, a membrane protein that is similar to MHC class I-type proteins and associates with beta-2 microglobulin. HFE regulates the interaction between the transferrin-receptor (TfR) with transferrin, the iron-transport protein in plasma. There are two transferrin receptors: TfR 1 is a transmembrane glycoprotein consisting of two disulfide-linked monomers joined by disulfide bonds. Each monomer binds one transferrin molecule resulting in an iron-Tf-TfR complex that enters the cell by endocytosis [6]. HFE was also shown to interact with TfR2 following an unproductive complex with TfR1. TfR2 is a membrane protein featuring a protease domain which mediates cellular uptake of diferric transferrin. However, HFE-TfR2 binding has not been confirmed in vivo and the interaction remains controversial [5]. Moreover, $\mathrm{Hfe}$ gene-deleted mice also exhibit faulty regulation of hepcidin expression as they develop iron overload $[5,7]$.

A more severe juvenile form of hemochromatosis is caused by mutations in a member of the three-gene family of repulsive guidance molecules, termed hemojuvelin $(H J V)$. HJV is a GPI-anchored membrane protein that interacts with bone morphogenetic proteins BMP2 and BMP4 [8]. The proteins could 
Fig. 1 Regulation of hepcidin expression in the liver. The ironsensing process involves transferrin-bound iron binding to TfR 1 causing a dissociation of HFE from the HFE/TfR1 partnership, possible relocation of HFE to TfR2 and the potential formation of a complex composed of Hfe/TfR2/Hjv and BMPR2 and BMPR1. The membrane complex subsequently activates phosphorylation of the Smad1/5/8 and subsequent binding of common Smad4, which directly activates hepcidin transcription. As it has been previously shown that combined deficiency of HFE and TfR2 results in decreased Erk signaling [11], additional or parallel signaling pathway to Bmp/Smad may be involved in the control of hepatic hepcidin transcription (adapted from [5])

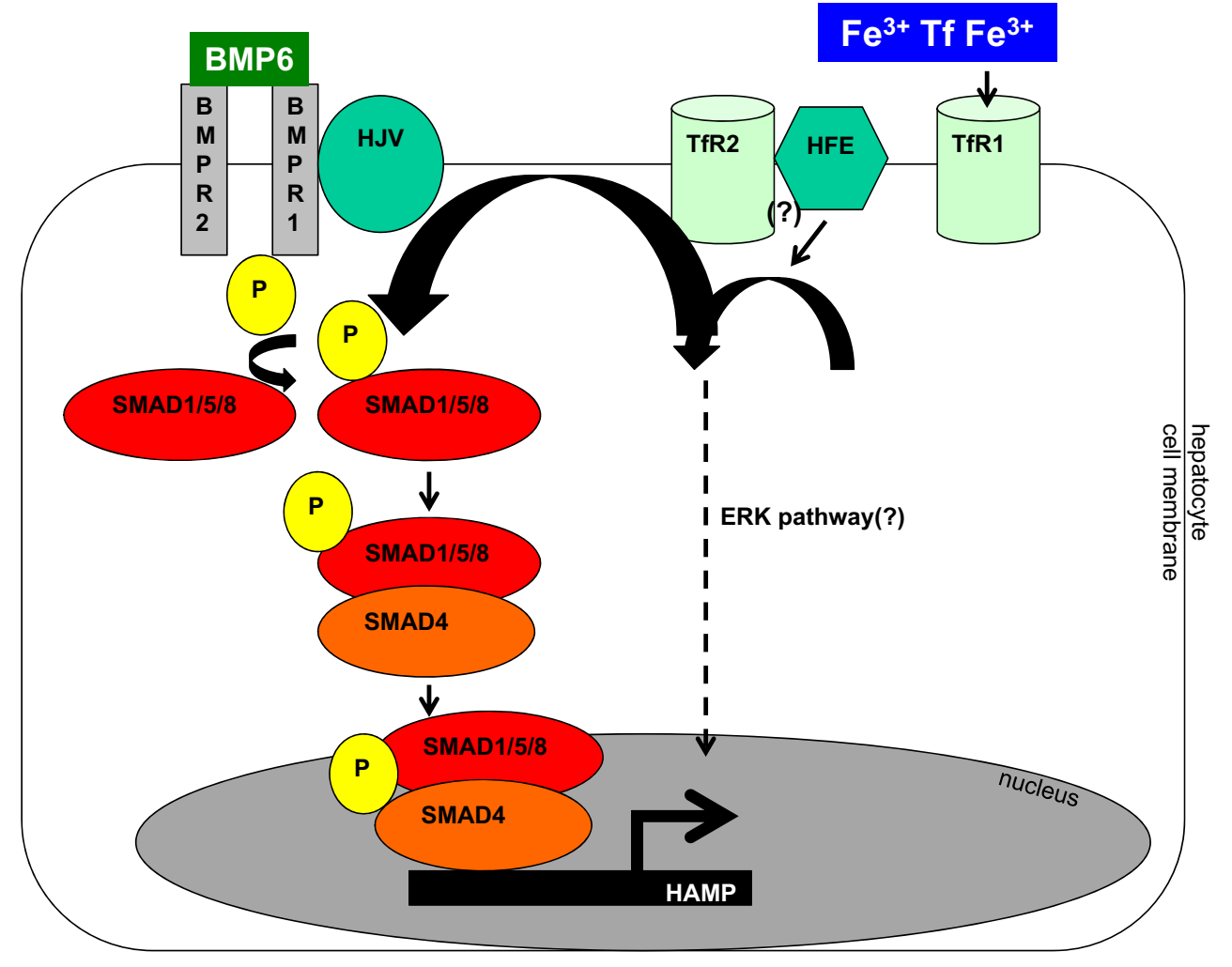

function as a co-receptor and signal via SMAD pathway to regulate hepcidin expression. Wu et al. recently suggested that HJV and HFE play distinct roles in regulating hepcidin [9]. They investigated Hfe and Hjv gene-deficient mice. The strains were then crossed to produce double-knockout mice. They found that the double-knockout mice had the same severe phenotype as $H j v$-deficient mice. Wu et al. concluded that HFE may depend on HJV for transferrin-dependent hepcidin regulation. They identified the presence of residual hepcidin in the absence of HFE and suggest that either the presence of an unknown regulator (for example, TfR2) that is synergistic with HJV or that HJV could be sufficient to maintain basal levels of hepcidin in their models [9].

In this issue of $J \mathrm{Mol} \mathrm{Med}$, Kent et al. also argue that HFE and HJV display overlapping functions to regulate hepcidin [10]. They also investigated $H f e$ and $H j v$ gene-deficient mice. The strains were then crossed to produce double-knockout mice. All the available genotypes were investigated regarding iron, iron-regulatory elements, and hepcidin expression in the serum, liver, and spleen. The investigators studied Smad signaling in the liver. As expected, the Hfe and Hjv gene-deleted mice developed hemochromatosis and more severe hemochromatosis, respectively, corresponding to the degree of hepcidin inhibition. The double-knockout mice resembled the $H j v$ gene-deleted mice phenotypically and in terms of hepcidin suppression. The authors suggest that $H f e$ and $H j v$ genes lead to an overlapping function in signaling to expression of hepcidin. Wu et al. did not cite Kent et al. and neither did Kent et al. cite Wu et al.; they could not because both papers were not yet published at the time of this writing [9, $10]$. Do the papers differ? Kent et al. fed their models a standard rodent diet ( $225 \mathrm{mg}$ iron per $\mathrm{kg}$ ) or alternatively the standard died supplemented with $2 \%$ carbonyl iron for 4 weeks in iron-loading experiments [10]. Wu et al. fed a similar standard diet and then relied on an iron-deficient diet for 3 weeks. Acute iron administration was performed in some animals that were given $2 \mathrm{mg}$ elemental iron as iron sulfate by gavage [9]. The findings are similar. It would take an iron chancellor to seek out details, an activity better left to experts. Future studies will eventually reveal the potential of this genetic model for deciphering precisely how HFE contributes to BMP-SMAD signaling in iron homeostasis (Fig. 1).

$$
\begin{aligned}
& \text { Respectfully, } \\
& \text { Friedrich C. Luft }
\end{aligned}
$$

\section{References}

1. Helms RA (2006) Textbook of therapeutics: drug and disease management, 8th edn. Lippincott Williams \& Wilkins, United States, Philadelphia

2. Przybyszewska J, Zekanowska E (2014) The role of hepcidin, ferroportin, HCP1, and DMT1 protein in iron absorption in the human digestive tract. Prz Gastroenterol 9:208-213

3. Gulec S, Collins JF (2014) Molecular mediators governing ironcopper interactions. Annu Rev Nutr 34:95-116 
4. Waldvogel-Abramowski S, Waeber G, Gassner C, Buser A, Frey BM, Favrat B, Tissot JD (2014) Physiology of iron metabolism. Transfus Med Hemother 41:213-221

5. Vujic M (2014) Molecular basis of HFE-hemochromatosis. Front Pharmacol 5:42

6. Franchini M (2006) Hereditary iron overload: update on pathophysiology, diagnosis, and treatment. Am J Hematol 81:202-209

7. Fleming RE, Feng Q, Britton RS (2011) Knockout mouse models of iron homeostasis. Annu Rev Nutr 31:117-137

8. Parrow NL, Fleming RE (2014) Bone morphogenetic proteins as regulators of iron metabolism. Annu Rev Nutr 34:77-94
9. Wu Q, Wang H, An P, Tao Y, Deng J, Zhang Z, Shen Y, Chen C, Min J, Wang F (2015) HJV and HFE play distinct roles in regulating hepcidin. Antioxid Redox Signal. doi:10.1089/ars.2013.5819

10. Kent P, Wilkinson N, Constante M, Fillebeen C, Gkouvatsos K, Wagner J, Buffler M, Becker C, Schumann K, Santos MM et al (2015) Hfe and Hjv exhibit overlapping functions for iron signaling to hepcidin. J Mol Med (Berl). doi:10.1007/s00109-015-1253-7

11. Wallace DF, Summerville L, Crampton EM, Frazer DM, Anderson GJ, Subramaniam VN (2009) Combined deletion of Hfe and transferrin receptor 2 in mice leads to marked dysregulation of hepcidin and iron overload. Hepatology 50:1992-2000 